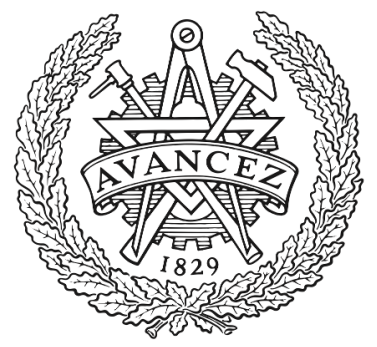

CHALMERS

UNIVERSITY OF TECHNOLOGY

\title{
Dark exciton based strain sensing in tungsten-based transition metal dichalcogenides
}

Downloaded from: https://research.chalmers.se, 2023-04-26 11:02 UTC

Citation for the original published paper (version of record):

Feierabend, M., Khatibi, Z., Berghäuser, G. et al (2019). Dark exciton based strain sensing in tungsten-based transition metal dichalcogenides. Physical Review B, 99(19).

http://dx.doi.org/10.1103/PhysRevB.99.195454

N.B. When citing this work, cite the original published paper. 


\title{
Dark exciton based strain sensing in tungsten-based transition metal dichalcogenides
}

\author{
Maja Feierabend, ${ }^{1}$ Zahra Khatibi, ${ }^{1,2}$ Gunnar Berghäuser, ${ }^{1}$ and Ermin Malic ${ }^{1}$ \\ ${ }^{1}$ Department of Physics, Chalmers University of Technology, 41296 Gothenburg, Sweden \\ ${ }^{2}$ Department of Physics, Iran University of Science and Technology, Narmak, 16846-13114, Tehran, Iran
}

(Received 10 January 2019; revised manuscript received 3 May 2019; published 29 May 2019)

\begin{abstract}
The recent emergence of atomically thin two-dimensional (2D) materials has opened up possibilities for the design of ultrathin and flexible nanoelectronic devices. As truly 2D materials, they exhibit an optimal surfaceto-volume ratio, which results in an extremely high sensitivity to external changes which can not be achieved by conventional semiconductors. This makes these materials optimal candidates for sensing applications. Here, we propose a dark exciton based concept for ultrasensitive strain sensors. By investigating both dark and bright excitons in tungsten-based monolayer transition metal dichalcogenides, we demonstrate that the dark-brightexciton separation can be controlled by strain, which has a crucial impact on the activation of dark excitonic states. The predicted opposite strain-induced shifts for dark and bright excitons result in a pronounced change in photoluminescence stemming from dark excitons even at very small strain values. The predicted high optical gauge factors of up to 8000 are promising for the design of optical strain sensors.
\end{abstract}

DOI: 10.1103/PhysRevB.99.195454

\section{INTRODUCTION}

Currently, the most common type of strain sensors consists of a metallic foil pattern, which becomes deformed in presence of strain resulting in a change of the electrical resistance [1-3]. In the case of small strain values, piezoresistors are more appropriate [4-6] since they have a larger gauge factor that is typically in the range of 10-20. The latter is defined as the ratio between the relative change in the electrical resistance and the applied mechanical strain. However, they are also more sensitive to temperature changes and are more fragile than foil-based sensors. The recently discovered class of atomically thin nanomaterials including graphene and monolayer transition metal dichalcogenides (TMDs) have the potential for the design of novel strain sensing devices [7,8]. Consisting of a single layer of atoms, they have an optimal surface-to-volume ratio resulting in an extremely high sensitivity to external stimuli. First, graphene-based strain sensors show large gauge factors in the range of 35-500 [9-11]. Values reported so far for $\mathrm{MoS}_{2}$ are in the range 40-140 [8,12], while the recently studied $\mathrm{PtSe}_{2}$ monolayers reveal a gauge factor of 85 [13]. However, the mentioned sensors are all based on a change in resistance due to strain, which makes them also sensitive to change in temperature or humidity.

In this work, we investigate another possible mechanism for sensing of strain based on dark excitons in twodimensional (2D) materials. TMD monolayers exhibit a remarkably rich exciton physics due to the strong Coulomb interaction leading to binding energies in the range of a few hundreds of meV. Aside from bright excitons, where both electron and hole are located in the $K$ valley with the same spin, the unique band structure of TMDs gives rise to the formation of dark excitons. Note that we focus here on tungstenbased TMDs since dark excitons can lie energetically below the bright excitons in these materials. We distinguish between spin-forbidden dark excitons consisting of electrons and holes with opposite spin and momentum-forbidden states with the Coulomb-bound electron and holes located in different valleys [14-21]. The latter cannot be directly accessed by light due to the required spin flip or large momentum transfer. In previous studies, it was shown that that the spin-forbidden states
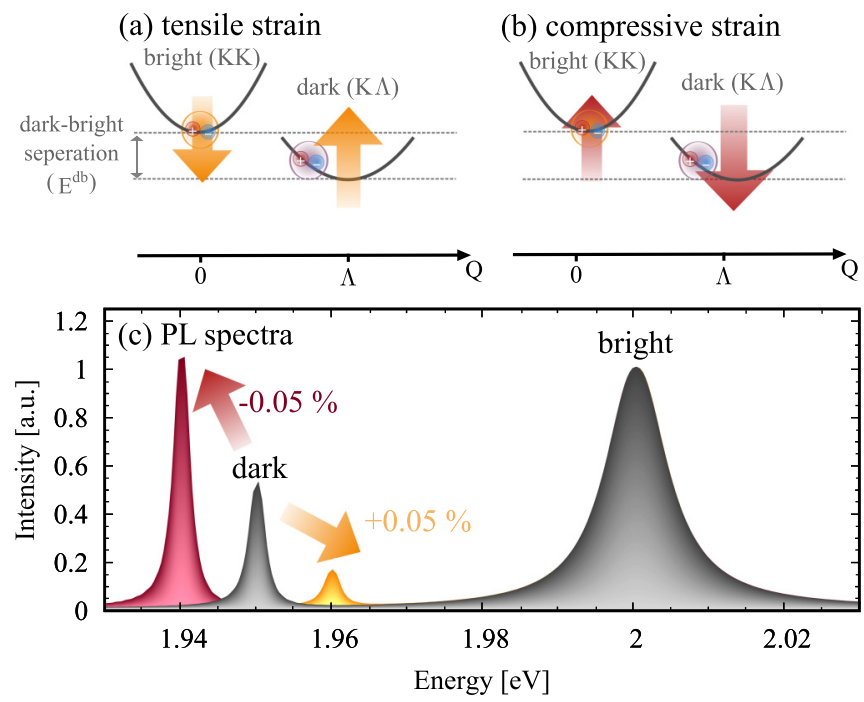

FIG. 1. Impact of strain on exciton dispersion and PL. (a) Tensile and (b) compressive strain shifts bright and momentum-forbidden dark exciton states in opposite direction. As a result, the spectral dark-bright separation $E^{\mathrm{db}}$ is strongly sensitive to strain. (c) Photoluminescence (PL) spectra for monolayer $\mathrm{WS}_{2}$ including the bright resonance at $2.0 \mathrm{eV}$ and the phonon-, disorder-, or molecule-activated dark resonance at $1.95 \mathrm{eV}$ in the unstrained case (gray line). Applying $0.05 \%$ tensile (compressive) strain leads to a blue-shift (red-shift) and a strong decrease (increase) in PL intensity of the dark exciton peak. This results in an optical gauge factor of 800 for the tensile and 8000 for the compressive strain. Note that the position of the bright exciton has been kept constant to focus on relative changes. 
consisting of Coulomb-bound electrons and holes with opposite spin can be brightened up either by applying strong inplane magnetic fields $[22,23]$ or via coupling to surface plasmons [24]. Momentum-forbidden states, where the Coulombbound electrons and holes are located in different valleys $(K$, $\Lambda)$ in the momentum space, can be activated through interaction with phonons [25] or high-dipole molecules [26,27] providing the required momentum to reach these states. Here, we focus on momentum dark excitons, where the Coulombbound electron and hole are located in different valleys. The state is denoted as dark exciton from now on. We show that the spectral separation of bright and momentum-forbidden dark excitonic states can be sensitively controlled by application of strain [Figs. 1(a) and 1(b)]. While tensile strain leads to a downshift (upshift) of the bright (dark) excitons in energy, compressive strain gives rise to an opposite behavior. Hence, strain can be used as an externally accessible tool to control the separation between dark and bright states in TMDs This has a huge impact on the photoluminescence (PL) intensity of activated dark states. A tensile strain of only $0.05 \%$ reduces the PL of the dark state by one order of magnitude [Fig. 1(c)]. This extremely high sensitivity (optical gauge factor of 800 for the tensile and 8000 for the compressive strain) suggests the possibility to design novel atomically thin dark exciton based strain sensors.

\section{THEORETICAL APPROACH}

To investigate strain-induced changes in the optical response of monolayer TMDs, we combine density functional theory (DFT) calculations providing access to the electronic band structure with density matrix formalism allowing us to calculate the excitonic binding energies and the excitonic photoluminescence. As a first step, we obtain the electronic structure of the unstrained monolayer tungsten disulfide $\left(\mathrm{WS}_{2}\right)$ that we investigate throughout the work as an exemplary TMD material. To this end, we relax the lattice structure using ultrasoft pseudopotential with local density approximation (LDA) exchange-correlation functionals alongside the plane waves implemented in the QUANTUM ESPRESSO package [28]. The momentum space is sampled with $18 \times 18 \times 1$ MonkhorstPack mesh and the kinetic energy cutoff is set to $60 \mathrm{Ry}$. The total forces on each atom after relaxation is less than $0.001 \frac{\mathrm{Ry}}{\mathrm{a} . u \text {. }}$. To avoid the possible interaction of adjacent layers, we use a vacuum space of approximately $20 \AA$ perpendicular to the $\mathrm{WS}_{2}$ layer. The calculations include spin-orbit coupling but the changes due to strain are significantly small. Hence, we will focus on the spin-up configuration from now on. For the unstrained case, we obtain a lattice constant of $a_{0}=$ $0.315 \mathrm{~nm}$, which is in good agreement with experimental values and previous DFT calculations [29]. In the next step, we model the strained $\mathrm{WS}_{2}$ monolayer via the change of the lattice constant relative to the strain with $a_{0}^{s}=a_{0}\left(1+\varepsilon_{s}\right)$. Here, $\varepsilon_{s}$ is the applied strain, while $a_{0}^{s}$ and $a_{0}$ denote the strained and unstrained lattice constant, respectively. Then, we relax the new lattice, while keeping the lattice vectors fixed at their strained value. Therefore, only atomic positions can relax with regard to the strained vectors. Note that we use the same energy mesh cutoff and $k$ grid for the strained $\mathrm{WS}_{2}$. Finally, we utilize non-self-consistent field calculation
TABLE I. Electronic and excitonic quantities under strain. Strain-induced changes in the electronic dispersion around the highsymmetry $K$ and $\Lambda$ points including the effective mass of the conduction band $m_{c}\left(m_{0}\right)$, the direct electronic gap $E^{K K}(\mathrm{eV})$, and the energetic difference between direct and the indirect electronic band gap $\Delta E^{K \Lambda}(\mathrm{eV})$. Interestingly, the direct and the indirect gaps shift in opposite direction under strain. The spectral position of the bright exciton $E_{\mathrm{exc}}^{K K}(\mathrm{eV})$ and the corresponding dark-bright splitting $E^{\mathrm{db}}=$ $E_{\mathrm{exc}}^{K \Lambda}-E_{\mathrm{exc}}^{K K}(\mathrm{eV})$ are obtained by evaluating the Wannier equation.

\begin{tabular}{lcrcccc}
\hline \hline Strain & $m_{c}^{K}$ & $m_{c}^{\Lambda}$ & $E^{K K}$ & $\Delta E^{K \Lambda}$ & $E_{\mathrm{exc}}^{K K}$ & $E^{\mathrm{db}}$ \\
\hline 0 & 0.26 & 0.5 & 2.4 & 0.050 & 2.0 & 0.050 \\
$\pm 1 \%$ & \pm 0.013 & \pm 0.0 & $\mp 0.14$ & \pm 0.19 & $\mp 0.12$ & \pm 0.19 \\
\hline \hline
\end{tabular}

to obtain the electronic dispersion for both relaxed unstrained and strained $\mathrm{WS}_{2}$ monolayers.

To investigate strain-induced changes in the optical response of monolayer TMDs, we use the density matrix formalism providing access to excitonic binding energies, wave functions, and photoluminescence [31,32]. We extract material-specific values as DFT input parameters for the solution of the Wannier equation (cf. Table I). This includes the curvature $m_{v(c)}^{K / \Lambda}$ of the valence $(v)$ and the conduction $(c)$ band at the high-symmetry $K$ and $\Lambda$ points as well as the direct electronic gap $E^{K K}$ between the conduction and the valence band with same spin at the $K$ point. The indirect electronic gap $E^{K \Lambda}$ can be expressed as $E^{K \Lambda}=E^{K K}+\Delta E^{K \Lambda}$ with $\Delta E_{K \Lambda}$ as the energy difference from the conduction band minimum at the $K$ and the $\Lambda$ points. In agreement with previous work $[33,34]$ we find that strain has a qualitatively different impact on the $K$ and the $\Lambda$ valleys : the conduction band minimum at the $K$ point shifts down (up) for tensile (compressive) strain compared to the fixed valence band maximum, whereas the conduction band minimum at the $\Lambda$ point shows the opposite behavior [cf. Fig. 2(a)]. Note that actually all band extrema show a downshift (upshift) for tensile (compressive) strain, however, the conduction band at the $K$ valley shifts stronger than that at the $\Lambda$ valley resulting in an opposite shift relative to the valence band. This can be understood as a consequence of the different orbital composition of the conduction band: while at the $K$ point it consists mainly of $d_{z^{2}}$ orbitals of the tungsten atoms and $p_{x}+p_{y}$ orbitals of the sulfur atoms, at the $\Lambda$ valley $d_{x y}, d_{x^{2}+y^{2}}$, and $d_{z^{2}}$ orbitals of the tungsten atoms play the major role $[35,36]$. When applying strain to the system, the distance between the atoms is changed and hence the overlap of the corresponding orbitals, leading to different strain rates in the shifts.

Note that the absolute position of dark and bright exciton states in the unstrained is still under debate. However, the reported relative changes due to strain are stable and agree to a large extent in different DFT studies [35,37,38]. The strain-induced changes in the electronic dispersion at highsymmetry points are also shown in Table I. For $\pm 1 \%$ tensile (compressive) strain, the $\mathrm{WS}_{2}$ monolayer is stretched (compressed) and the lattice constant is changed to $a_{0}=0.318 \mathrm{~nm}$ $\left(a_{0}=0.312 \mathrm{~nm}\right)$. For the unstrained case (gray line in Fig. 2), we find the direct band gap at the $K$ point. However, the minimum in the conduction band at the $\Lambda$ point is close in energy 

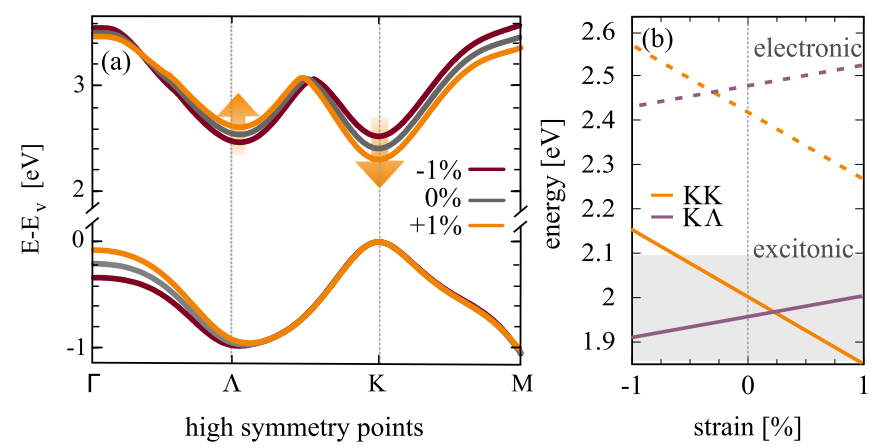

FIG. 2. Impact of strain on the electronic band structure. (a) DFT calculations of the electronic dispersion of the $\mathrm{WS}_{2}$ monolayer along the high-symmetry points in presence of tensile $(+1 \%)$ and compressive $(-1 \%)$ biaxial strain. To focus on relative changes, we use the valence band maximum at the $K$ point as the reference. We find that the conduction band at the $K$ point shifts down (up) by $150 \mathrm{meV}$ at $\pm 1 \%$ of tensile (compressive) strain. In contrast, the $\Lambda$ point shows the qualitatively opposite behavior resulting in an upshifts (downshifts) by $40 \mathrm{meV}$ for tensile (compressive) strain. Note that the absolute value of the band gap was adjusted to experimentally observed values [30]. (b) Strain-induced change of the energy of the bright $K K$ and the dark $K \Lambda$ excitons. While the first shows a decrease with strain (orange), the latter exhibits a positive slope resulting in a crossover at a certain strain value. The behavior can be mostly traced back to the strain dependence of the electronic $K$ and $\Lambda$ valleys. The dashed lines show the direct and indirect electronic gaps $E^{K K}$ and $E^{K \Lambda}$.

with $\Delta E^{K \Lambda}=50 \mathrm{meV}$. This distance is crucially affected by strain. When applying $1 \%$ tensile strain (yellow curve), the conduction band minimum at the $K$ point shifts down in energy by $-140 \mathrm{meV}$, while the conduction band minimum at the $\Lambda$ point shows an opposite behavior and shifts up by $50 \mathrm{meV}$ relative to the position of the valence band maximum at the $K$ point. For compressive strain (red curve), we observe the opposite behavior. As a result, we find a change of the dark-bright separation by $E^{K \Lambda}$ by $\pm 190 \mathrm{meV} / \%$, where + corresponds to the tensile and - to the compressive strain.

Regarding the curvature of the bands and hence the effective masses, we find only a relatively small change due to strain with $m_{c}^{K(\Lambda)}$ being modified by $2 \%$. The obtained values are in good agreement with previous DFT studies $[36,38]$. Note that we focus in this work on biaxial strain, which means that the lattice is deformed uniformly in all directions and thus strain can be modeled by changing the lattice constant. For uniaxial strain, our DFT calculation reveals a similar behavior regarding changes in energy shifts and effective masses. However, it is worth noting that in case of the uniaxial strain, the broken lattice symmetry is transferred to the orbital shape which can lead to a softening of valley-dependent optical selection rules. However, this effect is rather small [39] and thus $1 \%$ biaxial strain roughly corresponds to $2 \%$ uniaxial strain for the investigated range of relatively small strain values.

So far, we have discussed the impact of strain on the electronic properties of an exemplary TMD material. However, it is crucial to study the change of excitonic properties, which determine the optical response of these materials. In order to calculate optical spectra of TMDs including excitonic effects on a microscopic level, we solve the semiconductor Bloch equations, a coupled set of differential equations describing time- and momentum-resolved microscopic polarization, exciton occupation, as well as photon number [17,40-42]. To derive these equations of motion, we need to calculate the excitonic band structure as well as exciton-phonon and exciton-photon matrix elements. Thus, we solve the Wannier equation

$$
\frac{\hbar^{2} q^{2}}{2 m^{\mu}} \varphi_{\mathbf{q}}^{\mu}-\sum_{\mathbf{k}} V_{\mathrm{exc}}(\mathbf{k}) \varphi_{\mathbf{q}-\mathbf{k}}^{\mu}=\varepsilon_{\mathbf{b}}^{\mu} \varphi_{\mathbf{q}}^{\mu} .
$$

This is an eigenvalue equation for excitons with the excitonic eigenfunctions $\varphi_{\mathbf{q}}^{\mu}$ and the excitonic binding energies $\varepsilon_{b}^{\mu}$, where $\mu$ is the exciton index including in particular the $K K$ and the $K \Lambda$ excitons. Furthermore, $V_{\text {exc }}$ describes the Coulomb-induced formation of excitons within the Keldysh potential $[31,43]$. Here, we include the substrate via its dielectric screening constant and the screening length entering the Keldysh potential [44]. The exciton binding energy depends on the reduced exciton mass $m^{\mu}=\frac{m_{v}^{K} m_{c}^{\mu}}{m_{v}^{K}+m_{c}^{\mu}}$, where the conduction and valence band masses enter from our DFT calculations. For $\mathrm{WS}_{2}$ on $\mathrm{SiO}_{2}$ substrate, we obtain a binding energy of $\varepsilon^{K K}=400 \mathrm{meV}$ for the bright $K K$ exciton and $\varepsilon^{K \Lambda}=500 \mathrm{meV}$ for the dark $K \Lambda$ exciton. The latter is larger due to the heavier mass of the $\Lambda$ valley. Note that the spectral distance between dark and bright excitons can be tuned by dielectric screening [26]. Since strain has only a minor influence on the effective masses (Table I), the excitonic binding energy is only slightly affected by strain [39].

Our approach is consistent with previous experimental studies, which revealed strongly bound excitons with binding energies in the range of few hundreds of $\mathrm{meV}[45,46]$. Furthermore, the calculated excitonic linewidths in the range of tens of $\mathrm{meV}$ are in good agreement with recent experimental findings $[47,48]$ as well as the behavior of the linewidths under uniaxial strain [30]. Finally, the dynamics of excitons $[17,40,49]$ and the resulting temperature dependence of the photoluminescence yield as well as the dynamics of the intraexcitonic $1 s-2 p$ transition show an excellent agreement with experimental observations $[50,51]$.

The spectral position of the exciton can be expressed as $E_{\mathrm{exc}}^{K K}=E^{K K}-\varepsilon_{b}^{K K}$ and $E_{\mathrm{exc}}^{K \Lambda}=E^{K \Lambda}-\varepsilon_{b}^{K \Lambda}$. Note that the larger exciton binding energy of the $K \Lambda$ state exceeds the spectral differences in the electronic band structure between the $K$ and the $\Lambda$ valleys. As a result, the dark $K \Lambda$ exciton becomes lower in energy than the bright $K K$ state in tungstenbased TMDs [16,17]. We can write $E_{\mathrm{exc}}^{K \Lambda}=E_{\mathrm{exc}}^{K K}-E^{\mathrm{db}}$, where $E^{\mathrm{db}}$ corresponds to the spectral dark-bright-exciton separation [cf. Fig. 1(a)]. For the unstrained case, $E^{\mathrm{db}}=50 \mathrm{meV}$ in the $\mathrm{WS}_{2}$ monolayer, suggesting that this material is an indirectgap semiconductor in the excitonic picture.

To calculate the optical response of the system, we use the framework of the density matrix formalism in second quantization. The key quantity here is the photon number $n_{q}=\left\langle c_{\mathbf{q}}^{\dagger} c_{\mathbf{q}}\right\rangle$ with photon creation/annihilation operators $c_{\mathbf{q}}^{\dagger}$. The steady-state photoluminescence $\operatorname{PL}\left(\omega_{q}\right)$ is given by the rate of emitted photons $\operatorname{PL}\left(\omega_{q}\right) \propto \omega_{q} \frac{\partial}{\partial t}\left\langle c_{\mathbf{q}}^{\dagger} c_{\mathbf{q}}\right\rangle$, where $\omega_{q}$ is the photon frequency. Exploiting the Heisenberg equation of 
motion, we obtain for the dynamics of the photon number $\dot{n}_{q}=\left[n_{q}, H\right]$. The many-particle Hamilton operator $H$ includes the light-matter interaction describing the optical excitation of the system and the Coulomb interaction giving rise to the formation of strongly bound excitons. Furthermore, we include a mechanism for the activation of the momentumforbidden $K \Lambda$ excitons. This can be driven either by phonons, molecules, or actual disorder/impurity sites in the TMD lattice. In a recent PL study, indications for phonon-assisted radiative recombination pathways to momentum-forbidden excitons have been observed [52,53]. In previous theoretical work [26,27], we have discussed in detail the interaction of excitons with high-dipole molecules providing the required momentum to reach the dark $K \Lambda$ excitons.

The dynamics of the photon number $n_{q}$ is driven by photonassisted polarization [54] $S_{\mathbf{k}_{1} \mathbf{k}_{2}}^{v c_{\mu}}=\left\langle c_{\mathbf{q}}^{\dagger} a_{\mathbf{k}_{1}}^{\dagger v} a_{\mathbf{k}_{2}}^{c_{\mu}}\right\rangle$ with electron annihilation (creation) $a^{(\dagger)}$ and photon annihilation (creation) $c^{(\dagger)}$ operators. Solving the corresponding set of equations, we determine the steady-state photoluminescence on microscopic footing:

$$
I(\omega) \propto \operatorname{Im}\left(\frac{\left|M_{\omega}^{\sigma K}\right|^{2}}{\Delta E_{\omega}^{K K}-\frac{\left|G^{K \Lambda}\right|^{2}}{\Delta E_{\omega}^{K \Lambda}}}\left[N_{\mathbf{0}}^{K K}(1-\Gamma)+\Gamma N_{\mathbf{0}}^{K \Lambda}\right]\right)
$$

In the pristine case (i.e., $G^{K \Lambda}=0$ ), this leads to the wellknown Elliott formula in the incoherent limit. If $G^{K \Lambda} \neq 0$, i.e., in the case of a coupling to molecules, phonons, or disorder, we expect new dark excitons peaks to appear in the PL, whenever $\Delta E_{\omega}^{K \Lambda}=0$ Here, $M_{\omega}^{\sigma K}$ is the optical matrix element describing the optical excitation of the $K$ valley with the circularly polarized light $\sigma$. It determines the optical oscillator strength of excitonic resonances. The position of the latter in the PL spectrum is given by $\Delta E_{\omega}^{\mu}=E_{\mathrm{exc}}^{\mu}-\hbar \omega-i \gamma^{\mu}$ in the denominator. Finally, we have introduced an abbreviation $\Gamma=\frac{\left|G^{K \Lambda}\right|^{2}}{\left(\Delta E_{\omega}^{\Lambda}\right)\left(E^{\mathrm{db}}-i \gamma^{K \Lambda}\right)}$, where the matrix element $G^{K \Lambda}$ is given by the overlap of the excitonic wave functions $G_{\mathbf{Q k}}^{\mu \nu}=$ $\sum_{\mathbf{q}}\left(\varphi_{\mathbf{q}}^{\mu *} g_{\mathbf{q}_{\alpha}, \mathbf{q}_{\alpha}+\mathbf{k}}^{c c} \varphi_{\mathbf{q}+\beta \mathbf{k}}^{v}-\varphi_{\mathbf{q}}^{\mu *} g_{\mathbf{q}_{\beta}-\mathbf{k}, \mathbf{q}_{\beta}}^{v v} \varphi_{\mathbf{q}-\alpha \mathbf{k}}^{v}\right)$ with $\mathbf{q}_{\alpha}=\mathbf{q}-$ $\alpha \mathbf{Q}$ and $\mathbf{q}_{\beta}=\mathbf{q}+\beta \mathbf{Q}$. The exact form of the coupling element $g$ depends on the actual interaction that is necessary to activate the momentum-forbidden dark $K \Lambda$ excitonic state. This can i.e. driven by disorder, phonons, molecules, or any other momentum-generating mechanism. The particular mechanism is not important for this study.

We find that the PL is strongly influenced by exciton occupations $N_{\mathbf{0}}^{K K}, N_{\mathbf{0}}^{K \Lambda}$ with a vanishing center-of-mass momentum $\mathbf{Q} \approx \mathbf{0}$. Only these states are located in the light cone and can emit light through radiative recombination. After the process of phonon-induced exciton thermalization occurring on a timescale of 1 ps [17], the exciton occupations can be described by a Bose distribution. Figure 1(c) shows the calculated PL spectrum for unstrained $\mathrm{WS}_{2}$ monolayer at the exemplary temperature of $77 \mathrm{~K}$ (gray line). The peak at $2.0 \mathrm{eV}$ corresponds to the bright $K K$ exciton, while the energetically lower peak at $1.95 \mathrm{eV}$ stems from the dark $K \Lambda$ exciton. In the following section, we discuss in detail how these excitonic signatures in the PL spectrum change in presence of tensile and compressive strain.

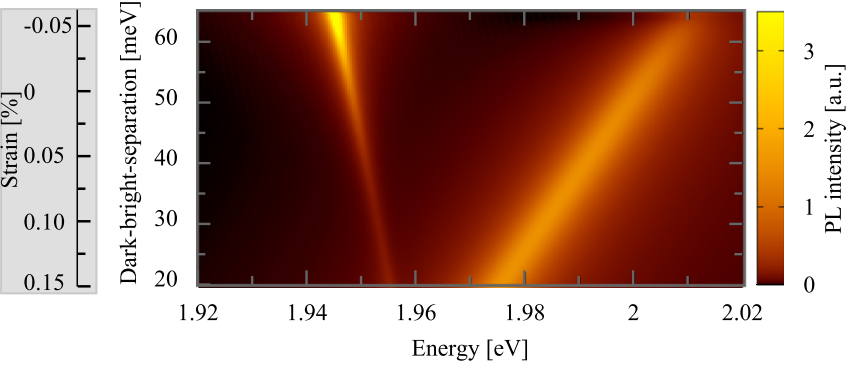

FIG. 3. Impact of strain on dark-bright-exciton separation and $\mathrm{PL}$ intensity. Spectrally resolved PL of the $\mathrm{WS}_{2}$ monolayer as a function of the dark-bright-exciton separation $E^{\mathrm{db}}$, which can be directly translated to a certain strain value. According to our calculations, the unstrained $\mathrm{WS}_{2}$ exhibits a dark-bright separation of $50 \mathrm{meV}$. Applying compressive strain (negative strain values), the energetic separation becomes larger, while for tensile strain (positive value) strain, it becomes smaller. We observe a pronounced change in the PL intensity of the dark $K \Lambda$, as it comes closer to the bright state.

\section{RESULTS}

Combining the DFT input for the strain-induced change of the electronic dispersion and the solution of the Wannier equation providing access to the excitonic binding energies, we calculate the exact spectral position of dark and bright excitonic states [cf. Fig. 2(b)]. While the energy of the bright $K K$ exciton decreases with tensile strain, the momentumforbidden dark $K \Lambda$ exciton shifts to higher energies. The strain-induced change can be fitted by a linear function with the slope $-145 \mathrm{meV} / \%$ for the bright and $+45 \mathrm{meV} / \%$ for the dark state. As a result, the spectral dark-bright separation $E^{\mathrm{db}}$ changes sign at $0.3 \%$ of tensile strain. This strongly influences the efficiency of the activation of dark $K \Lambda$ excitonic state and has a direct impact on its position and intensity in the PL spectrum. For comparison, the dashed lines in Fig. 2(b) visualize the direct and indirect electronic gaps $E^{K K}$ and $E^{K \Lambda}$ (without taking into account excitonic effects). We observe that the indirect gap lies energetically above the direct gap in the unstrained case. However, the strain behavior is qualitatively the same, reflecting the fact that the exciton binding energy is not very sensitive to strain [39]. As a result, it is the opposite strain dependence of the direct and indirect electronic gaps that determines the dark-brightexciton separation and thus also the spectral features and their strain sensitivity. Note that we do not include spin-forbidden dark states in our calculations since we find that the spin-orbit coupling is not significantly affected by strain, in agreement with previous studies [55]. Hence, this class of dark excitonic states shows the same strain behavior as bright excitons. Note that we have not investigated the possibility of strain-induced mixing of states with opposite spin, which could result in brightening of spin-forbidden dark excitonic states.

Figure 3 reveals the PL intensity of the $\mathrm{WS}_{2}$ monolayer as a function of energy and the dark-bright splitting (and strain) at the exemplary temperature of $77 \mathrm{~K}$. The broad peak at the higher-energy side stems from the bright exciton, shifting with a rate of $140 \mathrm{meV} / \%$ strain while the peak at the lower energy is ascribed to the dark $K \Lambda$ exciton that has been activated either via phonons, disorder, or molecules. The 
theoretical approach used in this paper has already shown very good agreement with experiment regarding both excitonic resonances and their linewidths $[25,31,41,47,56]$. The unstrained case corresponds to an energetic difference $E^{\mathrm{db}} \approx$ $50 \mathrm{meV}$ corresponding to the PL in Fig. 1(c). Interestingly, the smallest amounts of strain lead to pronounced changes in the optical response. This is due to the high sensitivity of the position and intensity of the dark exciton resonance to $E^{\mathrm{db}}$ that itself is highly sensitive to the applied strain. We predict a strain-induced change rate of $\pm 190 \mathrm{meV} / \%$ for $E^{\mathrm{db}}$ (Table I).

The strong change in the PL intensity of the dark exciton as the tensile strain increases and $E^{\mathrm{db}}$ decreases can be traced back to the change in the exciton population of the involved $K K$ and $K \Lambda$ excitons. The changes in the exciton occupation are directly reflected in the optical oscillator strength of the corresponding state. After the system is thermalized, the exciton population is given by the Bose distribution, where the energetic position of each excitonic state explicitly enters. If the material is tensile strained, the $K \Lambda$ exciton shifts up in energy, while the $K K$ exciton shifts down [Fig. 1(a)] resulting in a reduced dark-bright separation (Fig. 2). As a result, it becomes unfavorable for excitons to occupy the dark state. As a direct consequence, the intensity of the dark exciton is considerably reduced and the dark exciton can even be fully deactivated under certain strain values.

On the other hand, applying compressive strain shifts the dark exciton even lower in energy, resulting in a larger darkbright-exciton separation $E^{\mathrm{db}}$ and a higher occupation of the dark exciton state after thermalization. Hence, we predict the intensity of the dark exciton to significantly increase for compressive strain. Our calculations show that considerable changes in the optical response appear, when applying only $0.05 \%$ of strain (cf. Fig. 3). This suggests that smallest amounts of strain could be detected by measuring a simple PL spectrum. Note that as the TMD material is usually placed on a substrate, the strain is actually transferred from the substrate to the TMD layer. Depending on the transfer rate and the Poisson ratio of the substrate, the actual transferred strain to the TMD monolayer can be externally controlled. As a result, the extremely sensitive strain range depending on the TMD material and the used substrate can be shifted to the technologically desired values.

An important quantity for strain sensors is the gauge factor corresponding to the ratio between the relative strain-induced change in a certain property of the material and the actually applied mechanical strain. For our work, the optical gauge factor, defined as the ratio of the relative change in PL intensity and the strain, is a measure for the sensitivity of the dark exciton based strain sensor

$$
g_{x}=\frac{\Delta I_{x}[\%]}{\varepsilon_{s}[\%]}
$$

with the applied strain $\varepsilon_{s}[\%]$ and the intensity ratio $\Delta I_{x}=$ $\frac{I_{x}^{\mathrm{str}}-I_{x}^{0}}{I_{x}^{0}}$, where $x$ describes the bright $K K$ or the dark $K \Lambda$ exciton. Figure 4 shows the PL spectrum for unstrained and strained $\mathrm{WS}_{2}$ as well as strain-dependent intensity ratios and optical gauge factors. In addition to the bright (dark) gauge factor $g_{b}\left(g_{d}\right)$ that are determined by the strain-induced intensity changes of the bright (dark) $K K(K \Lambda)$ exciton, we
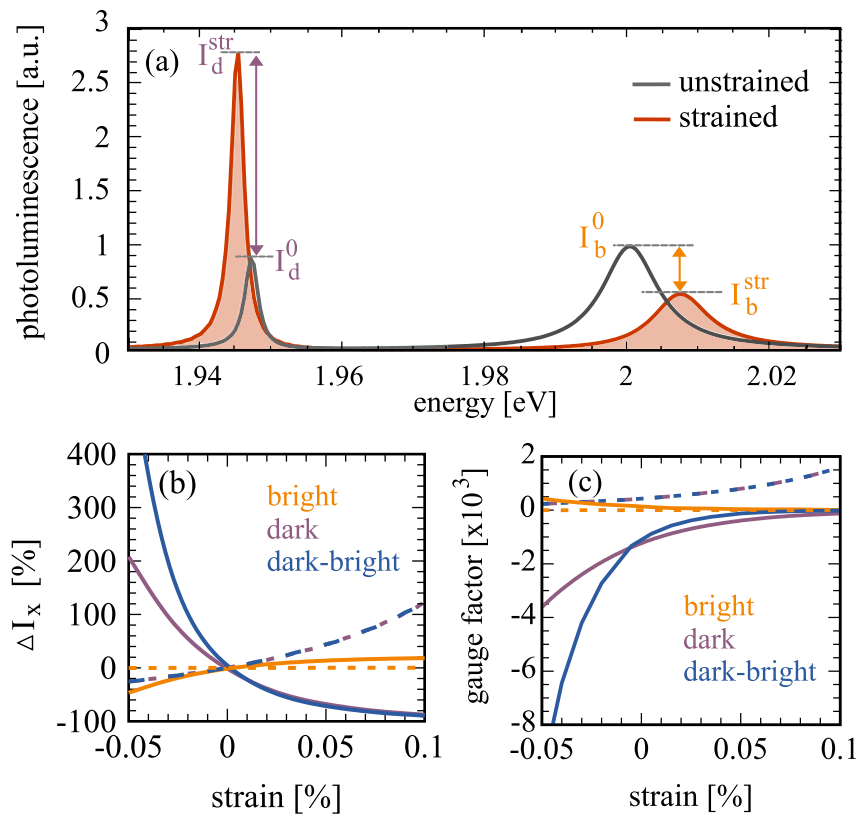

FIG. 4. Optical gauge factors. (a) PL spectrum of $\mathrm{WS}_{2}$ for the unstrained and the unstrained $(-0.05 \%)$ cases at $77 \mathrm{~K}$. Note that here the strain-induced shift and intensity change of the bright exciton is also shown. The extracted (b) intensity changes and (c) optical gauge factors $g_{x}$, where $x=b, d$ denote the bright $K K$ and the dark $K \Lambda$ exciton, respectively. The gauge factors are based on the intensity ratio between the unstrained $\left(I_{x}^{0}\right)$ and the strained $\left(I_{x}^{\text {str }}\right)$ cases for the dark exciton $g_{d}$ (purple) and the bright exciton $g_{b}$ (yellow) [cf. Eq. (3)]. We also show the corresponding gauge factor based on the change of the dark-bright-exciton ratio in presence of strain (blue). Dashed lines show the behavior at room temperature.

also consider the dark-bright gauge factor $g_{\mathrm{db}}$ that is based on the change of the intensity ratio between the dark and the bright excitons, i.e., $g_{\mathrm{db}}=\frac{I_{d}^{\mathrm{st}} / I_{b}^{\mathrm{str}}}{I_{d}^{0} / I_{b}^{0}}-1$. Figures $4(\mathrm{~b})$ and $4(\mathrm{c})$ show the corresponding changes in the relative intensity ratio $\Delta I_{x}$ and the gauge factors $g_{x}$ as a function of strain at $77 \mathrm{~K}$ (solid lines) and $300 \mathrm{~K}$ (dashed lines). In the case of a linear strain dependence, the gauge factors simply correspond to the slope of the corresponding lines. However, since the intensity changes are clearly nonlinear, the gauge factors vary with strain and can be understood as the derivative of the relative intensity ratio.

Our calculations reveal that one can obtain remarkably high gauge factors for the compressive strain at $77 \mathrm{~K}$. The dark-bright gauge factor $g_{\mathrm{db}}$ can reach values of up to 8000 for $0.05 \%$ of compressive strain (blue line). The dark gauge factor $g_{d}$ (purple line) goes up to 4000 , while the bright gauge factor $g_{b}$ (yellow curve) is in the range of 500. These values exceed reported values for $2 \mathrm{D}$ materials by an order of magnitude (35-500 for graphene [9-11], 40-140 for $\mathrm{MoS}_{2}$ [8,12], 85 for PtSe2 [13]). At room temperature (dashed lines), the gauge factors become lower in general, however, they are still high for $g_{d}$ and $g_{\mathrm{db}}$ reaching values up to 1500 , whereas $g_{b}$ considerably decreases to roughly 10 . As a result, at room temperature the dark gauge factors are two orders of magnitude higher than the bright gauge factor, opening up possible applications for ultrasensitive sensing of strain. 
To understand the high sensitivity of the dark exciton toward strain, we recapture its photoluminescence properties [cf. Eq. (2)]. The PL intensity is determined by exciton occupations $N^{x}$ that are given by the Bose distribution in equilibrium, where in particular $N^{K \Lambda} \propto N^{K K} \exp \left[-E^{\mathrm{db}}\left(k_{b} T\right)^{-1}\right]$ with the spectral bright-dark separation $E^{\mathrm{db}}$. As a result, we find for the strained intensity $I^{\mathrm{str}} \propto \exp \left[-E_{\mathrm{str}}^{\mathrm{db}}\left(k_{b} T\right)^{-1}\right]$, i.e., there is an exponential decrease of the PL intensity with strain, as observed in Fig. 4(b). For compressive (tensile) strain, $E^{\mathrm{db}}$ increases (decreases) and hence the dark state is stronger (weaker) occupied, resulting in an enhanced (reduced) PL intensity. At the same time, the bright exciton shows the opposite behavior since its occupation increases or decreases depending on the relative position of the dark state [cf. Figs. 4(b) and 4(c)]. As a result, we find remarkably high dark-bright gauge factor $g_{\mathrm{db}}$ in presence of compressive strain, where the strain-induced changes of the dark and the bright excitons add up, i.e., the PL intensity of the dark exciton increases and at the same time the intensity of the bright peak decreases. For tensile strain, the behavior is opposite since the dark peak becomes smaller, while the bright is enhanced resulting in $g_{\mathrm{db}}<g_{d}$ [cf. purple and blue lines in Fig. 4(c)].

For tensile strain values larger than $0.1 \%$, we find that all gauge factors go asymptotically toward zero at $77 \mathrm{~K}$. In presence of tensile strain, dark and bright excitons come closer together (Fig. 3) and the corresponding peaks start to spectrally overlap in the PL spectrum. Moreover, the dark exciton becomes less occupied, as it is higher in energy and hence disappears at some point. Note that for compressive strain, a saturation is also reached at higher strain values, reflecting the saturation of the dark exciton state. At room temperature [dashed lines in Figs. 4(b) and 4(c)] the behavior is different due to the much larger phonon-induced broadening of excitonic resonances. Here, the spectral overlap of the dark and the bright peaks becomes more important than the exciton occupation. The closer the states become, the higher is the PL intensity of the dark peak. As a result, in presence of tensile strain, larger dark and dark-bright gauge factors are predicted.

Our work presents an initial step toward the realization of strain sensors based on TMD materials. Using a microscopic approach, we demonstrate a proof of principle for strain sensors based on the optical response of dark excitonic states in these materials. Future studies are needed to reach a better visibility of dark excitons even at room temperature. Although the optical spectra in TMDs are characterized by clearly pronounced excitonic transitions, homogeneous and inhomogeneous broadening of these resonances (in particular at room temperature) as well as the spectral overlap of momentum-forbidden dark excitonic states with trions and/or defect excitons makes it challenging to trace back the features of single resonances. Note that we focus on the steady-state photoluminescence, where the assumption of a thermalized Bose distribution for excitons is justified. At the initial times of the exciton dynamics, high effective exciton temperatures will have an important impact on the quantitative value of the calculated gauge factors and need further investigation.

So far, we have studied and discussed the strain dependence in a $\mathrm{WS}_{2}$ monolayer as an exemplary TMD material. We expect a comparable behavior for $\mathrm{WSe}_{2}$, as it shows a similar electronic and excitonic dispersion, in particular with respect to the relative position of the dark $K \Lambda$ exciton [16]. Aside from the tungsten-based TMDs with the dark $K \Lambda$ excitons, also the molybdenum-based TMDs can show dark excitons. However, the case of $\mathrm{MoS}_{2}$ is a bit more complicated since here the energetically lowest $\Gamma K$ exciton needs to be taken into account. Since this exciton requires a rather large momentum transfer to be activated, we expect $\mathrm{MoS}_{2}$ to be less appropriate for strain sensing. Finally, $\mathrm{MoSe}_{2}$ is the only direct-gap semiconductor in the excitonic picture [16], where all indirect excitons lie above the bright state. It still could be used for strain sensing, as strain can move dark and bright states closer to each other and enable the activation of dark excitons in PL spectra. A summary of strain-dependent excitonic energies of $K K, K \Lambda$, and $\Gamma K$ excitons in all four TMDs can be found in our previous work [57].

\section{CONCLUSION}

In conclusion, we have presented a microscopic study revealing the proof of principle for strain sensors based on dark excitons in atomically thin 2D materials. In particular, we have investigated the strain-induced control of the optical response in a $\mathrm{WS}_{2}$ monolayer as an exemplary material for transition metal dichalcogenides. We have focused on the change of the photoluminescence intensity of bright and activated dark excitonic states. We find that the changes in the electronic band structure play the crucial role since they determine the spectral separation of dark and bright excitonic states. We predict a remarkable sensitivity to strain resulting in optical gauge factors in the range of up to 8000 . The obtained insights are the first step toward ultrasensitive strain sensing based on dark excitons in atomically thin materials.

\section{ACKNOWLEDGMENTS}

This project has received funding from the European Union's Horizon 2020 research and innovation programme under Grant Agreement No. 785219. Furthermore, we acknowledge financial support from the Swedish Research Council (VR).
[1] J. Zhou, Y. Gu, P. Fei, W. Mai, Y. Gao, R. Yang, G. Bao, and Z. L. Wang, Nano Lett. 8, 3035 (2008).

[2] X. Wang, J. Zhou, J. Song, J. Liu, N. Xu, and Z. L. Wang, Nano Lett. 6, 2768 (2006).

[3] J. Sirohi and I. Chopra, J. Intell. Mater. Syst. Struct. 11, 246 (2000).
[4] C. W. Hicks, M. E. Barber, S. D. Edkins, D. O. Brodsky, and A. P. Mackenzie, Rev. Sci. Instrum. 85, 065003 (2014).

[5] S. Kon, K. Oldham, and R. Horowitz, in Sensors and Smart Structures Technologies for Civil, Mechanical, and Aerospace Systems 2007, Vol. 6529 (SPIE, Bellingham, WA, 2007), p. $65292 \mathrm{~V}$. 
[6] J.-U. Youn, Y.-W. Choo, and D.-S. Kim, Can. Geotech. J. 45, 1426 (2008).

[7] C. S. Boland, U. Khan, C. Backes, A. O'Neill, J. McCauley, S. Duane, R. Shanker, Y. Liu, I. Jurewicz, A. B. Dalton et al., ACS Nano 8, 8819 (2014).

[8] M.-Y. Tsai, A. Tarasov, Z. R. Hesabi, H. Taghinejad, P. M. Campbell, C. A. Joiner, A. Adibi, and E. M. Vogel, ACS Appl. Mater. Interfaces 7, 12850 (2015).

[9] C. Casiraghi, M. Macucci, K. Parvez, R. Worsley, Y. Shin, F. Bronte, C. Borri, M. Paggi, and G. Fiori, Carbon 129, 462 (2018).

[10] X. Li, R. Zhang, W. Yu, K. Wang, J. Wei, D. Wu, A. Cao, Z. Li, Y. Cheng, Q. Zheng et al., Sci. Rep. 2, 870 (2012).

[11] J. Zhao, C. He, R. Yang, Z. Shi, M. Cheng, W. Yang, G. Xie, D. Wang, D. Shi, and G. Zhang, Appl. Phys. Lett. 101, 063112 (2012).

[12] M. Park, Y. J. Park, X. Chen, Y.-K. Park, M.-S. Kim, and J.-H. Ahn, Adv. Mater. 28, 2556 (2016).

[13] S. Wagner, C. Yim, N. McEvoy, S. Kataria, V. Yokaribas, A. Kuc, S. Pindl, C.-P. Fritzen, T. Heine, G. S. Duesberg et al., Nano Lett. 18, 3738 (2018).

[14] A. Arora, M. Koperski, K. Nogajewski, J. Marcus, C. Faugeras, and M. Potemski, Nanoscale 7, 10421 (2015).

[15] G. Wang, C. Robert, A. Suslu, B. Chen, S. Yang, S. Alamdari, I. C. Gerber, T. Amand, X. Marie, S. Tongay et al., Nat. Commun. 6, 10110 (2015).

[16] E. Malic, M. Selig, M. Feierabend, S. Brem, D. Christiansen, F. Wendler, A. Knorr, and G. Berghäuser, Phys. Rev. Mater. 2, 014002 (2018).

[17] M. Selig, G. Berghäuser, M. Richter, R. Bratschitsch, A. Knorr, and E. Malic, 2D Mater. 5, 035017 (2018).

[18] W.-T. Hsu, L.-S. Lu, D. Wang, J.-K. Huang, M.-Y. Li, T.-R. Chang, Y.-C. Chou, Z.-Y. Juang, H.-T. Jeng, L.-J. Li et al., Nat. Commun. 8, 929 (2017).

[19] T. Deilmann and K. S. Thygesen, Phys. Rev. B 96, 201113(R) (2017).

[20] T. Mueller and E. Malic, 2D Mater. Appl. 2, 29 (2018).

[21] G. Wang, C. Robert, M. M. Glazov, F. Cadiz, E. Courtade, T. Amand, D. Lagarde, T. Taniguchi, K. Watanabe, B. Urbaszek, and X. Marie, Phys. Rev. Lett. 119, 047401 (2017).

[22] A. V. Stier, N. P. Wilson, G. Clark, X. Xu, and S. A. Crooker, Nano Lett. 16, 7054 (2016).

[23] M. Molas, C. Faugeras, A. Slobodeniuk, K. Nogajewski, M. Bartos, D. Basko, and M. Potemski, 2D Mater. 4, 021003 (2017).

[24] Y. Zhou, G. Scuri, D. S. Wild, A. A. High, A. Dibos, L. A. Jauregui, C. Shu, K. De Greve, K. Pistunova, A. Y. Joe et al., Nat. Nanotechnol. 12, 856 (2017).

[25] D. Christiansen, M. Selig, G. Berghäuser, R. Schmidt, I. Niehues, R. Schneider, A. Arora, S. M. de Vasconcellos, R. Bratschitsch, E. Malic et al., Phys. Rev. Lett. 119, 187402 (2017).

[26] M. Feierabend, G. Berghäuser, A. Knorr, and E. Malic, Nat. Commun. 8, 14776 (2017).

[27] M. Feierabend, G. Berghäuser, M. Selig, S. Brem, T. Shegai, S. Eigler, and E. Malic, Phys. Rev. Mater. 2, 014004 (2018).

[28] P. Giannozzi, S. Baroni, N. Bonini, M. Calandra, R. Car, C. Cavazzoni, D. Ceresoli, G. L. Chiarotti, M. Cococcioni, I. Dabo et al., J. Phys.: Condens. Matter 21, 395502 (2009).
[29] A. Kormanyos, G. Burkard, M. Gmitra, J. Fabian, V. Zolyomi, N. D. Drummond, and V. Falko, 2D Mater. 2, 022001 (2015).

[30] I. Niehues, R. Schmidt, M. Drüppel, P. Marauhn, D. Christiansen, M. Selig, G. Berghäuser, D. Wigger, R. Schneider, L. Braasch et al., Nano Lett. 18, 1751 (2018).

[31] G. Berghäuser and E. Malic, Phys. Rev. B 89, 125309 (2014).

[32] E. Malic, J. Maultzsch, S. Reich, and A. Knorr, Phys. Rev. B 82, 115439 (2010).

[33] A. Molina-Sanchez, K. Hummer, and L. Wirtz, Surf. Sci. Rep. 70, 554 (2015).

[34] S. Deng, L. Li, and M. Li, Phys. E (Amsterdam) 101, 44 (2018).

[35] C.-H. Chang, X. Fan, S.-H. Lin, and J.-L. Kuo, Phys. Rev. B 88, 195420 (2013).

[36] Q. Yue, J. Kang, Z. Shao, X. Zhang, S. Chang, G. Wang, S. Qin, and J. Li, Phys. Lett. A 376, 1166 (2012).

[37] B. Amin, T. P. Kaloni, and U. Schwingenschlögl, Rsc Adv. 4, 34561 (2014).

[38] H. Shi, H. Pan, Y.-W. Zhang, and B. I. Yakobson, Phys. Rev. B 87, 155304 (2013).

[39] M. Feierabend, A. Morlet, G. Berghäuser, and E. Malic, Phys. Rev. B 96, 045425 (2017).

[40] S. Brem, M. Selig, G. Berghaeuser, and E. Malic, Sci. Rep. 8, 8238 (2018).

[41] S. Brem, A. Ekman, D. Christiansen, F. Katsch, M. Selig, C. Robert, X. Marie, B. Urbaszek, A. Knorr, and E. Malic, arXiv:1904.04711.

[42] M. Hirtschulz, F. Milde, E. Malić, S. Butscher, C. Thomsen, S. Reich, and A. Knorr, Phys. Rev. B 77, 035403 (2008).

[43] L. V. Keldysh, Sov. J. Exp. Theor. Phys. Lett. 29, 658 (1979).

[44] M. Feierabend, E. Malic, A. Knorr, and G. Berghäuser, J. Phys.: Condens. Matter 29, 384003 (2017).

[45] M. M. Ugeda, A. J. Bradley, S.-F. Shi, H. Felipe, Y. Zhang, D. Y. Qiu, W. Ruan, S.-K. Mo, Z. Hussain, Z.-X. Shen et al., Nat. Mater. 13, 1091 (2014).

[46] K. He, N. Kumar, L. Zhao, Z. Wang, K. F. Mak, H. Zhao, and J. Shan, Phys. Rev. Lett. 113, 026803 (2014).

[47] M. Selig, G. Berghäuser, A. Raja, P. Nagler, C. Schüller, T. F. Heinz, T. Korn, A. Chernikov, E. Malic, and A. Knorr, Nat. Commun. 7, 13279 (2016).

[48] A. Singh, A. Knorr, C. K. Dass, C.-H. Chen, E. Malic, G. Moody, G. Clark, G. Berghäuser, K. Hao, K. Tran et al., Nat. Commun. 6, 8315 (2015).

[49] G. Berghäuser, P. Steinleitner, P. Merkl, R. Huber, A. Knorr, and E. Malic, Phys. Rev. B 98, 020301(R) (2018).

[50] X.-X. Zhang, Y. You, S. Y. F. Zhao, and T. F. Heinz, Phys. Rev. Lett. 115, 257403 (2015).

[51] P. Steinleitner, P. Merkl, A. Graf, P. Nagler, K. Watanabe, T. Taniguchi, J. Zipfel, C. Schüller, T. Korn, A. Chernikov et al., Nano Lett. 18, 1402 (2018).

[52] J. Lindlau, C. Robert, V. Funk, J. Förste, M. Förg, L. Colombier, A. Neumann, E. Courtade, S. Shree, T. Taniguchi et al., arXiv:1710.00988.

[53] J. Lindlau, M. Selig, A. Neumann, L. Colombier, J. Förste, V. Funk, M. Förg, J. Kim, G. Berghäuser, T. Taniguchi et al., Nat. Commun. 9, 2586 (2018). 
[54] A. Thränhardt, S. Kuckenburg, A. Knorr, T. Meier, and S. W. Koch, Phys. Rev. B 62, 2706 (2000).

[55] R. Roldán, J. A. Silva-Guillén, M. P. López-Sancho, F. Guinea, E. Cappelluti, and P. Ordejón, Ann. Phys. (NY) 526, 347 (2014).
[56] S. Brem, J. Zipfel, M. Selig, A. Raja, L. Waldecker, J. Ziegler, T. Taniguchi, K. Watanabe, A. Chernikov, and E. Malic, arXiv:1904.04729.

[57] Z. Khatibi, M. Feierabend, M. Selig, S. Brem, C. Linderälv, P. Erhart, and E. Malic, 2D Mater. 6, 015015 (2018). 